\title{
SIMULATING GRANULAR COLUMN COLLAPSE BY NON-SMOOTH CONTACT DYNAMICS
}

\author{
J. Huang ${ }^{1}$, K. Krabbenhoft ${ }^{1}$, M. Vicente da Silva ${ }^{1,2}$, A.V. Lyamin ${ }^{1}$
}

${ }^{1}$ Centre for Geotechnical Science and Engineering, University of Newcastle, Callaghan, NSW 2308, Australia (jinsong.huang@ newcastle.edu.au)

${ }^{2}$ UNIC, Department of Civil Engineering, Faculty of Sciences and Technology, Universidade Nova de Lisboa, Quinta da Torre, 2829-516 Monte da Caparica, Portugal

\begin{abstract}
We present $3 D$ numerical simulations of the collapse and spreading of granular columns based on non-smooth contact dynamics and conic programming. A novel rolling resistance model is developed which can model non-spherical particles using spheres at a lower computation cost. The simulated final heights and run-out distances are in good agreements with experimental studies.
\end{abstract}

Keywords: Non-smooth contact dynamics, Rolling resistance, Collapse of granular column

\section{INTRODUCTION}

The flow of granular media plays an important role in many different areas, including geotechnical engineering, agriculture, chemical engineering, the earth sciences, fundamental physics, hazard management, and the pharmaceutical industry. Partly because of this multidisciplinary nature there has been considerable research interest in this area recently. Consequently, granular flows have been the subject of numerous works, either theoretical, experimental or numerical. In the absence of a clear physical background for the modelling of these large-scale natural granular flows, their characterization relies mainly on the observation of the final deposits, and in particular, of the final run-out distance. A simple setting, in which an initially cylindrical column of granular material is suddenly released and allowed to collapse freely onto a horizontal plane, has drawn increased attentions in recent years. These studies shed some lights on some of the key questions - What governs the spread dynamics? What determines the final deposit geometry and run-out distance? The main result found by experimental studies (e.g., [1-6]) is the so called scaling laws for run-out distance. In particular, when the initial aspect ratio of the collapsing column is large enough, the run-out distance normalized by the initial radius of the column shows a power-law dependence on the initial aspect ratio. Moreover, this dependence varies with the conditions of the experiment, in particular between axisymmetric and quasi-two-dimensional configurations.

Numerical studies followed the experimental ones. The main object of those numerical studies is to reproduce the scaling laws observed by experiments. Commonly used methods 
can be classified into continuum and discrete approaches. Larrieu et al. [7] adopted a modified shallow water model successfully reproduced the scaling laws observed by Lube et. al [1] and Lajeunesse et al. [2]. Lacaze and Kerswell [8] did three dimensional simulations based on a viscoplastic continuum theory. These simulations faithfully reproduce the different flow regimes and capture the observed scaling laws for the final deposit. Three dimensional discrete element simulations were also conducted. Mangeney-Castelnau et al. [9] used continuum hydrodynamic models and their dimensional analysis showed that the final run-out distance depends only on the initial aspect ratio and effective friction angle.

Discrete approaches (e.g., [10-13]) mainly use classic discrete element method pioneered by Cundall and Strack [14]. Most recently, Krabbenhoft et al. [15] developed a variational approach to contact dynamics for simulation granular flow. The method is based on Non-Smooth Contact Dynamics Method developed by Moreau, Jean and their coworkers [16, 17]. The work by Krabbenhoft et al. [15] is extended to three dimension in this paper. The 3D numerical simulations of the collapse and spreading of granular columns are presented. A novel rolling resistance model is developed which can model non-spherical particles using spheres at a lower computation cost. The simulations faithfully reproduced the final heights and run-out distances observed by experimental studies.

\section{REVIEW ON THE EXPERIMENTAL RESULTS}

Experiments by Lube et al. [1] and Lajeunesse et al. [2] were performed by rapidly releasing granular materials, initially contained in a partially filled cylinder, on to a flat surface and allowing them to spread out unhindered. The main result consists of scaling laws for the run-out distance. Lube et al. [1] find

$$
\frac{r_{\infty}-r_{0}}{r_{0}}= \begin{cases}1.24 a, & 0 \leq a<1.7 \\ 1.6 a^{0.5}, & 1.7 \leq a<10\end{cases}
$$

where $a=h_{0} / r_{0}, r_{0}$ and $h_{0}$ are initial radius and height, $r_{\infty}$ and $h_{\infty}$ are final radius and height.

Lajeunesse et al. [2] find

$$
\frac{r_{\infty}-r_{0}}{r_{0}}= \begin{cases}1.35 a, & 0 \leq a<0.74 \\ 2.0 a^{0.5}, & 0.74 \leq a<10\end{cases}
$$

The scaling law for the normalized final height obtained by Lube et al. [1] is

$$
\frac{h_{\infty}}{r_{0}}= \begin{cases}a, & 0 \leq \mathrm{a}<1.0 \\ 0.88 a^{\frac{1}{6}}, & 1.0 \leq \mathrm{a}<10\end{cases}
$$

while Lajeunesse et al. [2] find

$$
\frac{h_{\infty}}{r_{0}}= \begin{cases}a, & \mathrm{a}<0.74 \\ 0.74 a, & \mathrm{a}>0.74\end{cases}
$$




\section{GRANULAR CONTACT DYNAMICS USING MATHMETICAL PROGRAMMING}

A series of concise mathematical programming formulations of granular contact dynamics have been developed, implemented and tested by Krabbenhoft et al. [15]. In contrast to traditional discrete element analysis, the granular contact dynamics formulation uses an implicit time discretization, thus allowing for large time steps. The approach leads naturally to a formulation in terms of mathematical programming as shown in Eq. (5) and Fig. 1. Interested readers are referred to [15] for detailed derivation.

$$
\begin{gathered}
\text { maximize }-\frac{1}{2} \mathbf{a}^{\mathrm{T}} \overline{\mathbf{M}}^{-1} \mathbf{a}-\frac{1}{2} \mathbf{t}^{\mathrm{T}} \overline{\mathbf{J}}^{-1} \mathbf{t}-\mathbf{g}_{0}^{\mathrm{T}} \mathbf{p} \\
\text { subject to } \mathbf{a}+\mathbf{p} \mathbf{N}_{0}+\mathbf{q}=\overline{\mathbf{f}}_{0} \\
\mathbf{t}-\mathbf{R} \mathbf{N}_{0} \times \mathbf{q}=\overline{\mathbf{m}}_{0} \\
\|\mathbf{q}\|-\mu \mathbf{p} \leq 0 \\
\mathbf{q N}_{0}=0
\end{gathered}
$$

where $\mathbf{a}$ is dynamic force related to translation, $\overline{\mathbf{M}}$ is normalized mass matrix, $\mathbf{t}$ is dynamic force related to rotation, $\overline{\mathbf{J}}$ is normalized mass moment of inertia matrix, $\mathbf{g}_{0}$ is gap between particles, $\mathbf{p}$ is normal contact force, $\mathbf{q}$ is shear contact force, $\mathbf{N}_{0}$ is contact normal direction, $\overline{\mathbf{f}}_{0}$ is normalized external force, $\overline{\mathbf{m}}_{\mathbf{0}}$ is normalized external moment, $\mathbf{R}$ is particle radius matrix, $\mu$ is sliding friction coefficient.

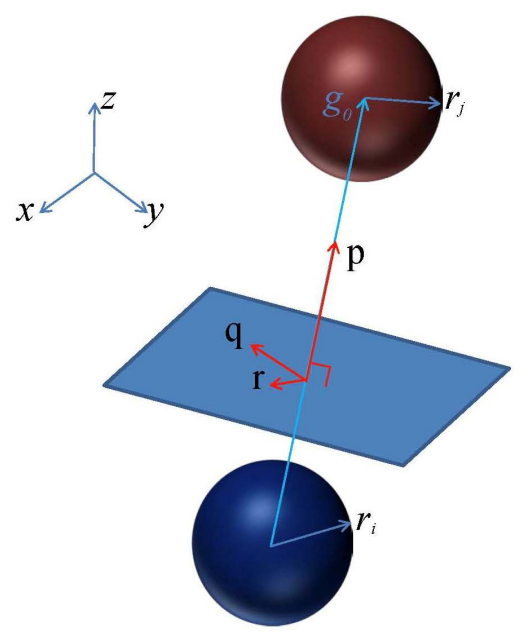

Fig. 1 Contact forces between particles

It was found however, using Eq. (5) to simulate collapse of granular column led to a final planar deposit. This is mainly because of spherical particles. Spheres have no rolling resistance but are computational efficient. In order to simulate the rolling resistance of irregular particles by spheres, a novel rolling resistance model is developed in the present study. The resulting mathematical programming formula is 


$$
\begin{array}{cc}
\text { maximize } & -\frac{1}{2} \mathbf{a}^{\mathrm{T}} \overline{\mathbf{M}}^{-1} \mathbf{a}-\frac{1}{2} \mathbf{t}^{\mathrm{T}} \overline{\mathbf{J}}^{-1} \mathbf{t}-\mathbf{g}_{0}^{\mathrm{T}} \mathbf{p} \\
\text { subject to } & \mathbf{a}+\mathbf{p} \mathbf{N}_{0}+\mathbf{q}=\overline{\mathbf{f}}_{0} \\
\mathbf{t}-\mathbf{R} \mathbf{N}_{0} \times \mathbf{q}=\overline{\mathbf{m}}_{\mathbf{0}}+\mathbf{R r} \\
\|\mathbf{q}\|-\mu \mathbf{p} \leq 0 \\
\|\mathbf{r}\|-\mu_{r} \mathbf{p} \leq 0 \\
\mathbf{q N} \mathbf{N}_{0}=0 \\
\mathbf{r N}_{0}=0
\end{array}
$$

where $\mathbf{r}$ is rolling resistance force and $\mu_{r}$ is rolling resistance coefficient.

As shown in Eq. (6) and Fig. 1, the rolling resistance is modelled in a way that is analogical to sliding friction. The governing equations were modified such that a moment above a certain threshold is required for rolling to commence. This approach is favored because only one new parameter $\mu_{r}$ is introduced.

Rolling resistance coefficient can be easily estimated. The principle is illustrated in Fig. 2 where a hexagonal particle with a weight amounting to a force $f$ is considered. In order to map the hexagon into an equivalent disk with radius $r$, it is necessary to establish a relation between the side length of the hexagon, $l$ and the radius of the disk. This can be done by assuming equal perimeters which leads to

$$
l=\frac{1}{3} \pi r
$$

In order for a hexagonal particle to roll, i.e. in order for the angle $\theta$ to increase above zero, a moment $\tau=1 / 2 p l$ is required. It is here evident that the rolling resistance is generated by the eccentricity of the contact force $p$. The moment necessary for rolling to commence is not constant during the course of a full revolution. Indeed, in the present case, the resisting moment attains its maximum value for $\theta=0^{\circ}$ and the decrease to become equal to zero at $\theta=60^{\circ}$ and remain zero until $\theta=120^{\circ}$. This suggests an average resisting moment of

$$
\tau_{\text {ave }}=\frac{1}{8} p l
$$

The resisting moment of a disk is constant during a full course of revolution. According to Eq. (6),

$$
\tau_{\text {disk }}=\mu_{r} r p
$$

Assuming $\tau_{\text {ave }}=\tau_{\text {disk }}$ and by Eq. (7),

$$
\mu_{r}=\frac{1}{24} \pi=0.131
$$




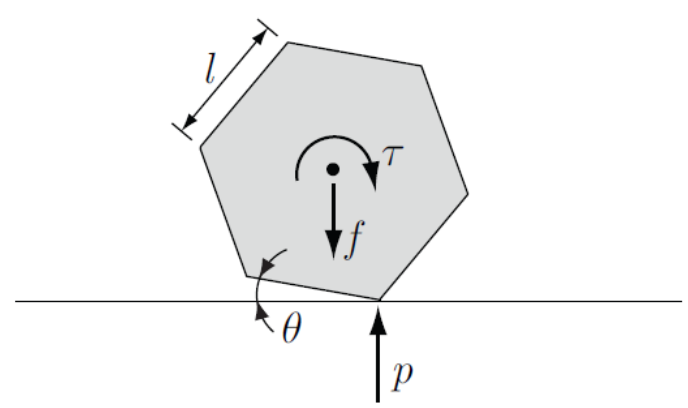

Fig. 2 Rolling resistance of two-dimensional hexagonal particle

The resulting programs (i.e., Eq. 6) can be solved using standard and readily available algorithms. In terms of implementation, the key operations comprise the assembly of system matrices similar to those of the finite element method. These system matrices define the mathematical program to be solved in each time step. The final, and crucial, task of solving these programs can be carried out using a general purpose solver - in 2D a quadratic programming solver and in 3D a second-order cone programming solver. Such solvers are becoming increasingly common and both commercial and open-source codes are readily available. In the present study, the academic version of Mosek was used.

\section{COLLAPSES OF GRANULAR COLUMNS}

The setup of our simulation is shown in Fig. 3. A column is initially filled by free falling particles created uniformly across the top of the cylinder and these are then allowed to settle. The top surface is then trimmed at the desired height. The particle diameters were uniformly distributed between 0.25 and 0.5 . Particles have a constant mass density of one. The sliding friction coefficient and rolling resistance coefficient are both set to zero in the filling stage. The mean solid fraction (relative packing density) is 0.58 , which coincides with the values observed in experiments. The columns then collapse under the influence of gravity. The simulations are carried out for a total time of $\quad \bar{t}=t / \sqrt{h_{0} / g}=4.0$ using 100 time steps of equal magnitude $\Delta \bar{t}=0.04$. The sliding friction coefficient and rolling resistance coefficient are set to 0.577 and 0.12 in all simulations.

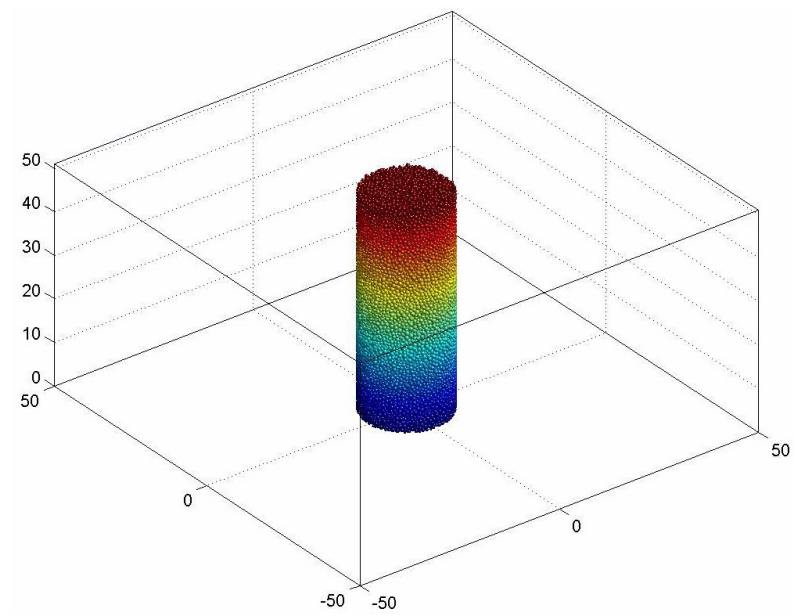

Fig. 3 Initial packing of a granular column 
Six granular columns with aspect ratios of $a=0.5,1.0,2.0,3.0,4.0$ and 5.0 were simulated. It is straight forward to define the final height. It is not obvious, how the run-out distance is best defined. In the present study, the final run-out distance is defined by the outmost grain that is connected to the main mass by at least one contact. The definition of final height $h_{\infty}$ and run-out distance $r_{\infty}$ are shown in Fig. 4.

For flows with $a<1.0$, a circular undisturbed area at the upper surface of the column, which remains at $h_{0}$, is preserved as shown in Fig. 4. In contrast, for columns with $a>1.0$, flow erodes the entire initial column surface leaving a conical pile at the peak (Fig. 5). The critical aspect ratio that separates the scaling laws for final height is believed to be $a=1.0$ as shown in Eq. (1). The simulated final heights were compared with experimental results by Lube et al. [1] in Fig. 6.

For flows with $a>1.7$, the entire upper surface starts to flow immediately. This is the critical aspect ratio that separates the scaling laws for final run-out distance as described in Eq. (1). The simulated final run-out distances were compared with experimental results by Lube et al. [1] in Fig. 7.

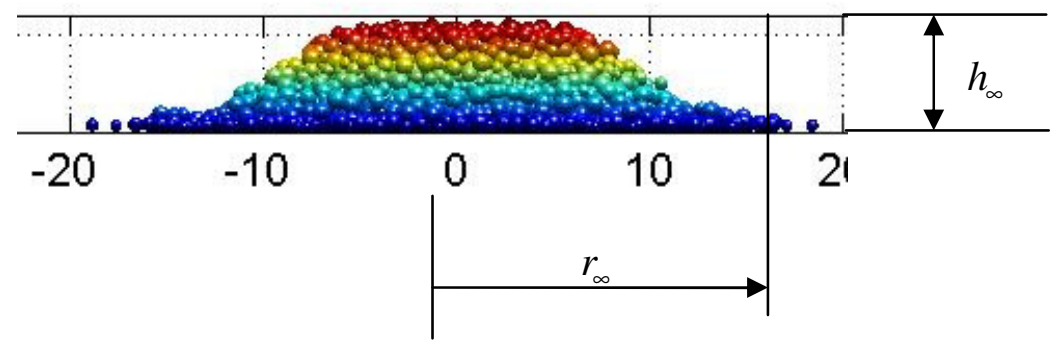

Fig. 4 Final height and run-out distance $(a=0.5)$

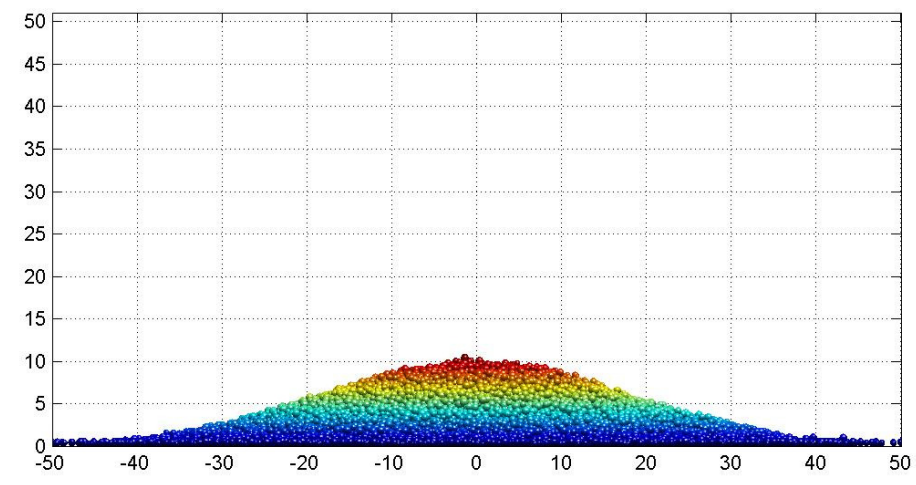

Fig. 5 Final deposit for $a=5.0$ 


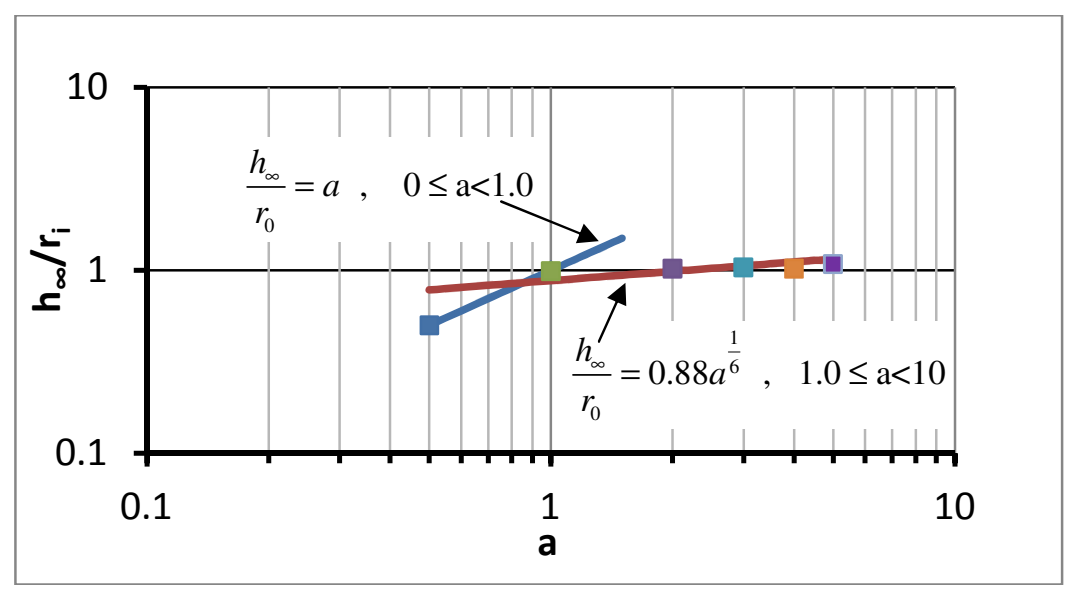

Fig. 6 Final heights compared with experiments

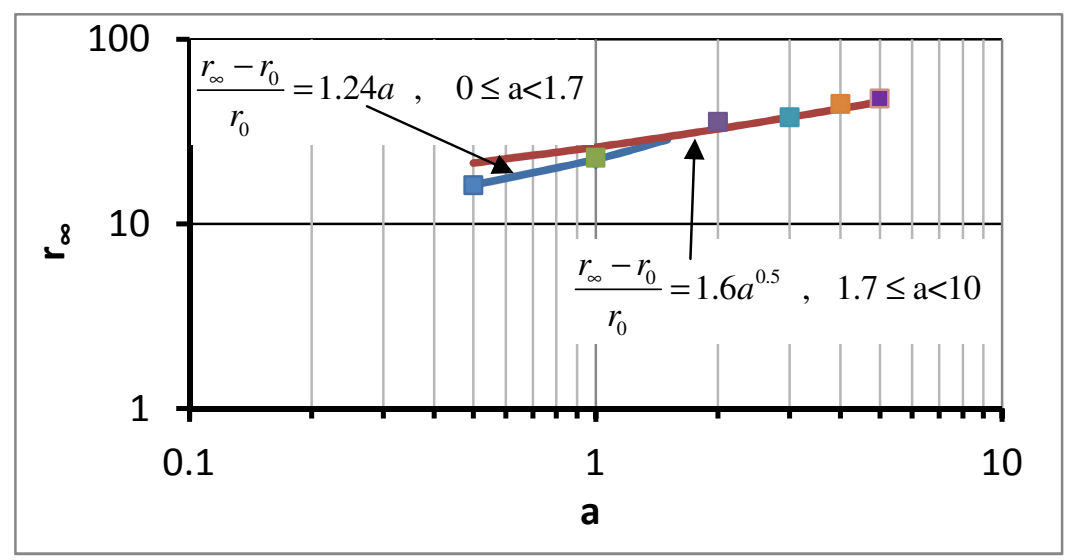

Fig. 7 Final run-out distances compared with experiments

\section{CONCLUSIONS}

The application of non-smooth contact dynamics and conic programming to simulate granular flow is presented. A novel rolling resistance model is developed which can model non-spherical particles using spheres at a lower computation cost. The developed model is validated by comparisons with experiments of the collapse of axisymmetric granular columns as reported in the literature. Excellent agreement is observed between the model simulations and experimental observations with regard to the final height and run-out distance. This study indicates that the developed model can be used to effectively simulate granular flows such as landslides and debris flow.

\section{Acknowledgements}

The work described in this paper was supported by the Australian Research Council Centre of Excellence for Geotechnical Science and Engineering, for which the authors are very grateful. 